\title{
Elevated circulating macrophage inhibitory cytokine 1 is a biological marker for the diagnosis and prognosis of osteosarcoma
}

\author{
XIANXIANG SONG, LIJUN TIAN, YANQIU QUAN, DAN SUN and LINMEI ZHANG \\ Clinical Laboratory, Xuzhou Children's Hospital, Xuzhou, Jiangsu 221006, P.R. China
}

Received December 20, 2017; Accepted July 6, 2018

DOI: $10.3892 /$ etm.2018.6786

\begin{abstract}
Osteosarcoma is among the most frequently occurring bone tumors in infants and teenagers. However, despite its widespread prevalence, no effective diagnostic and prognostic biomarkers for osteosarcoma are known. Macrophage inhibitory cytokine 1 (MIC-1) has been considered a promising biological marker of various tumor types. However, the possible role of circulating MIC-1 as a screening biomarker for osteosarcoma remains to be elucidated. The present study evaluated the circulating levels of MIC-1 in patients with osteosarcoma with the aim of elucidating its effect on the diagnosis and prognosis of this specific tumor. The circulating levels of MIC-1 were measured via an enzyme-linked immunosorbent assay in 300 individuals, including 100 patients with osteosarcoma, 100 patients with benign bone tumors, and 100 healthy subjects, and its correlation with clinicopathological characteristics was then evaluated. Various analyses were performed to determine its utility in diagnosis and prognosis. The levels of circulating MIC-1 were increased considerably in patients with osteosarcoma. The patients bearing larger tumors, those with distant metastases, and those with later-stage tumors had relatively higher levels of MIC-1. According to the multivariate logistic regression analysis, a high level of circulating MIC-1 was an independent variable for distant metastases. Receiver operating characteristic analysis revealed MIC-1 as a possible biological marker for distinguishing patients from healthy controls. Patients with osteosarcoma with higher levels of MIC-1 had relatively higher risk of mortality. Furthermore, multivariate data analysis on general survival rate revealed that a high level of circulating MIC-1 was a prognostic indicator of osteosarcoma. These findings suggest that an elevated level of circulating MIC-1 is a novel potential diagnostic and prognostic biomarker for osteosarcoma.
\end{abstract}

Correspondence to: Dr Xianxiang Song, Clinical Laboratory, Xuzhou Children's Hospital, 18 Sudi North Road, Xuzhou, Jiangsu 221006, P.R. China

E-mail: xxsong1029@163.com

Key words: biological marker, macrophage inhibitory cytokine 1, osteosarcoma, prognosis, diagnosis

\section{Introduction}

Osteosarcoma is one of the most frequently diagnosed primary malignant bone tumors in children and teenagers, with an incidence rate of $\sim 4-5$ individuals in every $1,000,000$ individuals $(1,2)$. It originates from osteoid production and nurtures immature bone tissue (3). Despite the emergence and advances in various novel therapies, including adjuvant chemotherapy or radiotherapy, wide local excision of relative regions is still observed, with significant morbidity and mortality rates among patients with osteosarcoma $(4,5)$. The five-year overall survival rate and five-year disease-free survival rate of osteosarcoma have been reported to be $\sim 50-60$, and $\sim 40 \%$ of patients with osteosarcoma eventually succumb to mortality from metastases in the lung tissue (6-8). Consequently, it is imperative to develop novel diagnostic strategies for osteosarcoma at early phases to provide more therapeutic alternatives. Elucidating the underlying molecular pathogenesis of osteosarcoma is important as it can assist in targeting diagnostic and prognostic biomarkers for the most effective therapeutic approach.

Macrophage inhibitory cytokine 1 (MIC-1) is a secretory protein of the transforming growth factor- $\beta$ (TGF- $\beta$ ) family and is important in carcinogenesis-related processes, including diffusion, metastasis, apoptosis and revascularization; furthermore, it is involved in irregular immune activities (9-11). MIC-1 has been considered as a potential candidate biomarker of colorectal carcinoma $(12,13)$. Currently, comprehensive confirmation regarding the association between circulating MIC-1 levels and early diagnosis of osteosarcoma is required.

In the present study, circulating MIC-1 levels were measured via an enzyme-linked immunosorbent assay (ELISA), following which its correlation with tumor properties was assessed, and the possibility of its use as a novel potential diagnostic and prognostic maker for osteosarcoma was examined.

\section{Materials and methods}

Participants. In total, 300 patients were included in the present study, which included 100 patients with primary osteosarcoma who were treated in Xuzhou Children's Hospital (Xuzhou, China) between March 2012 and May 2015. Osteosarcoma was diagnosed via clinical and microanatomical examination, and 
by histological assessment of specimens from osteosarcoma tissue. Participants did not have any history of other tumors and did not undergo any other pre-treatment when the initial diagnosis of osteosarcoma was confirmed. To select patients with benign bone tumors, 100 patients with benign bone tumors, including osteoenchondroma, ecchondrosis, bone cysts and fibrous osteoma, were included. These bone-related benign tumors were diagnosed via histological examination, similar to osteosarcoma. An additional 100 healthy age- and gender-matched subjects constituted the control group. These individuals were not diagnosed with any orthopedic issue or tumor. The experiments and all follow-up evaluations gained approval from the Ethics Committee of Xuzhou Children's Hospital (no. 20130116) and all participants provided written informed consent.

Data recording and follow-up evaluations. For each patient, his/her demographic information, tumor site, metastasis position, and pathological pattern were recorded upon diagnosis. All patients with osteosarcoma were treated with standard therapeutic pro Ethics Committee of cesses involving new accessorial chemotherapy and wide-margin surgical excision coupled with adjuvant chemotherapy or radiotherapy. Chemotherapy-induced reactions were categorized into two types on the basis of histological assessment of the osteosarcoma specimens: Poor, when tumor cell necrosis rate was $<90 \%$; good, when tumor cell necrosis rate was $>90 \%$. Furthermore, all patients with osteosarcoma were monitored postoperatively. The continuous disease-free survival period was recorded from the date of surgery until the time of tumor recurrence or the patient succumbed to mortality. The final follow-up record was on May 2017 and the median value of overall survival was 40.1 months in a total range of 10-75 months.

ELISA for circulating MIC-1. Venous blood samples from patients with osteosarcoma were obtained upon diagnoses. The blood samples were stored in serum separator tubes for coagulation, followed by centrifugation at a speed of $7500 \mathrm{xg}$ for $20 \mathrm{~min}$ at $4^{\circ} \mathrm{C}$ to separate serum into other tubes at $-80^{\circ} \mathrm{C}$ prior to ELISA for circulating MIC-1. The level of circulating MIC-1 was assessed via a sensitive in-house ELISA (RAB0204; Sigma-Aldrich; EMD Millipore, Billerica, MA, USA). Remixed MIC-1 (50 $\mu \mathrm{l})$ in different concentrations and serum were added in a 96-well plate, with wells coated with $5 \mu \mathrm{g} / \mathrm{ml}$ anti-MIC-1 antibody. Thereafter, $50 \mu \mathrm{l}$ of $0.2 \mu \mathrm{g} / \mathrm{ml}$ rabbit anti-MIC-1 biotinylated antibody was mixed in and incubated for $1 \mathrm{~h}$ at $37^{\circ} \mathrm{C}$. Streptavidin-HRP was added following cleaning the plates with washing buffer (cat. no. RAB0204; Sigma-Aldrich; Merck KGaA, Darmstadt, Germany), followed by incubation for $30 \mathrm{~min}$ at $37^{\circ} \mathrm{C}$. Eventually, the optical density of every well was measured on a microplate reader at $450 \mathrm{~nm}$. Based on the standard curve plotted from stepwise dilution of the recombinant MIC-1, circulating MIC-1 levels from each individual were calculated. All samples were assayed in duplicate.

Reverse transcription-quantitative polymerase chain reaction (RT-qPCR) analysis. Resected osteosarcoma tissue samples and non-cancer bone tissues were obtained and incubated at $-80^{\circ} \mathrm{C}$. RNA was isolated from the frozen tissues using TRIzol (Invitrogen; Thermo Fisher Scientific, USA), in accordance with the manufacturer's protocol. Total RNA ( $1 \mu \mathrm{g}$ of every sample) was used for cDNA synthesis, using a reverse transcription kit (Takara Bio, Inc., Otsu, Japan). Subsequently, RT-qPCR analysis was performed for synthesized cDNA under the following cycling conditions: $2 \mathrm{~min}$ pre-treatment at $95^{\circ} \mathrm{C}$, followed by $60^{\circ} \mathrm{C}$ for $15 \mathrm{sec}, 72^{\circ} \mathrm{C}$ and $95^{\circ} \mathrm{C}$ each for $30 \mathrm{sec}$, in $35 \mathrm{cycles}$, and extension at $72^{\circ} \mathrm{C}$ for $5 \mathrm{~min}$. The samples were then maintained at $4^{\circ} \mathrm{C}$. RT-qPCR reactions were performed using an ABI Prism 7000 sequence detection system (Applied Biosystems; Thermo Fisher Scientific, Inc., Waltham, MA, USA) with the GoTaq qPCR Master Mix (Promega Corporation, Madison, WI, USA). The following primers were used: MIC-1, forward 5'-CGGAATTCATGG CGCGCAACG-3' and reverse 5'-CCCTCGAGTATGCAG TGGCAGT-3'; $\beta$-actin, forward 5'-AGGCACCAGGGCGTG AT-3' and reverse 5'-GCCCACATAGGAATCCTTCTG AC-3'. Relative mRNA levels of all genes were normalized against the levels of $\beta$-actin using the $\Delta \Delta \mathrm{Cq}$ method (14). The experiments were repeated three times.

Western blot analysis. The tumor tissues and their adjacent tissues were homogenized in ice and lysed in RIPA buffer with protease inhibitors (Sigma; EMD Millipore) and phenylmethylsulfonyl fluoride (Sigma; EMD Millipore). The dissolved solid was sonicated and centrifuged at a speed of $7,500 \mathrm{x} \mathrm{g}$ at $4^{\circ} \mathrm{C}$ for $5 \mathrm{~min}$. Supernatant liquor was obtained following precipitation. Proteins were extracted their concentrations were determined using a BCA Protein kit (Thermo Fisher Scientific, Inc.). Protein $(50 \mu \mathrm{g})$ was then separated via sodium dodecyl sulfate polyacrylamide gel electrophoresis (10\% resolving gel) and subsequently electroblotted onto polyvinylidene fluoride membranes (EMD Millipore), which were washed with Tris-buffered saline and $0.05 \%$ Tween 20 , followed by blocking with $5 \%$ skimmed milk. The membrane was then incubated with anti-MIC-1 (Thermo Fisher Scientific, Inc.; cat. no. PA5-71579, 1:1,000) and $\beta$-actin antibodies (Santa Cruz Biotechnology, Inc., Dallas, TX, USA, cat. no. sc-130656, 1:1,000) at $4^{\circ} \mathrm{C}$ overnight. The membrane was then incubated with HRP-conjugated secondary antibody (Santa Cruz Biotechnology, Inc., cat. no. sc-2004, 1:4,000) at room temperature for $1 \mathrm{~h}$. Following washing, protein bands were detected using an Enhanced Chemiluminescence Western Blotting kit (Thermo Fisher Scientific, Inc.) and then quantified by Image J 1.43 software (National Institutes of Health, Bethesda, MD, USA).

Statistical analysis. One-way analysis of variance was performed to determine differences in circulating MIC-1 levels in the osteosarcoma patient group, benign tumor group, and control group. A t-test was performed to quantify the mRNA expression of MIC-1 in tumor tissues and associated peri-carcinomatous tissue. Diagnostic efficiency was evaluated via receiver operating characteristic (ROC) analysis. Survival rates were determined via Kaplan-Meier survival analysis. Statistically significant differences were measured via the log-rank test. Multivariable assessment of factors affecting prognosis was performed via Cox's regression analysis model. Statistical analysis was performed using Prism 5 software 
Table I. Demographics of the study population.

\begin{tabular}{lcccrr}
\hline Factor & $\begin{array}{c}\text { Total } \\
(\mathrm{n}=300)\end{array}$ & $\begin{array}{c}\text { Osteosarcoma } \\
(\mathrm{n}=100)\end{array}$ & $\begin{array}{c}\text { Benign tumor } \\
(\mathrm{n}=100)\end{array}$ & $\begin{array}{c}\text { Healthy } \\
(\mathrm{n}=100)\end{array}$ & P-value \\
\hline Male gender & $193(64.3)$ & $59(59)$ & $70(70)$ & $64(64)$ & 0.32 \\
Age (years) & $13.3(8.21)$ & $12.2(10.22)$ & $13.1(9.27)$ & $13.8(8.93)$ & 0.62 \\
BMI $\left(\mathrm{kg} / \mathrm{m}^{2}\right)$ & $23.02(1.45)$ & $23.59(1.54)$ & $23.01(1.34)$ & $22.66(1.43)$ & 0.45 \\
\hline
\end{tabular}

Values are expressed as $\mathrm{n}(\%)$ or mean \pm standard deviation. BMI, body mass index.

(GraphPad Software, Inc., La Jolla, CA, USA). P<0.05 was considered to indicate a statistically significant difference.

\section{Results}

Clinical features and circulating MIC-1 levels. The present study investigated the association between circulating levels of MIC-1 and clinicopathological characteristics in patients with osteosarcoma. The clinical features of the samples are shown in Tables I and II. There were no considerable differences among patient features. As shown in Fig. 1, the circulating MIC-1 levels were elevated in patients with osteosarcoma compared with those in the benign bone tumor group and the healthy controls. In addition, the levels of MIC-1 in patients with benign tumors were marginally, higher than those of the healthy controls.

Correlation between circulating MIC-1 levels and clinicopathological features of patients with osteosarcoma. The present study investigated the correlation between the level of circulating MIC-1 and clinicopathological features in patients with osteosarcoma. The levels of circulating MIC-1 did not correlate with age, gender, or chemotherapeutic response. Patients with osteosarcoma with thigh bone tumors had a marginally, but not significantly, higher level of circulating MIC-1 than those with tumors at other locations (data not shown). The analysis of tumor size indicated that circulating MIC-1 levels were increased significantly in tumors $>8 \mathrm{~cm}$, compared with those that were $<8 \mathrm{~cm}(\mathrm{P}<0.05$; Fig. 2A). Circulating MIC-1 levels differed among patients at various clinical stages; patients with stage III tumors had a significantly higher level of circulating MIC-1 than those with stage IIA and IIB tumors ( $\mathrm{P}<0.05$ for both). Similarly, as shown in Fig. 2B, patients with stage IIB tumors had significantly higher levels of circulating MIC-1 than those with stage IIA osteosarcoma $(\mathrm{P}<0.05)$. Circulating MIC-1 was higher in patients with osteosarcoma with distant metastases and in low tumor grades $(\mathrm{P}<0.05$; Fig. $2 \mathrm{C}$ and $\mathrm{D})$.

ROC analysis of circulating MIC-1 in patients with osteosarcoma. Assessment of the area under the ROC curves (ROC/AUC) determined the sensitivity and specificity of circulating $\mathrm{MIC}-1$ at different concentrations. Circulating expression levels of MIC-1 were considered a biological indicator for distinguishing patients with osteosarcoma from healthy controls (Fig. 3A). This measurement may also assist in differentiating between patients with osteosarcoma and
Table II. Characteristics of the study population.

\begin{tabular}{|c|c|c|c|}
\hline Characteristics & $\begin{array}{l}\text { Osteosarcoma } \\
\quad(n=100)\end{array}$ & $\begin{array}{l}\text { Benign tumor } \\
\quad(\mathrm{n}=100)\end{array}$ & $\begin{array}{l}\text { Healthy } \\
(n=100)\end{array}$ \\
\hline \multicolumn{4}{|l|}{ Age (years) } \\
\hline$\leq 20$ & 73 & 76 & 80 \\
\hline$>20$ & 27 & 24 & 20 \\
\hline \multicolumn{4}{|l|}{ Gender (n) } \\
\hline Male & 59 & 70 & 64 \\
\hline Female & 41 & 30 & 36 \\
\hline \multicolumn{4}{|l|}{ Location (n) } \\
\hline Femur & 69 & & \\
\hline Tibia & 29 & & \\
\hline Other & 12 & & \\
\hline \multicolumn{4}{|l|}{ Size $(\mathrm{cm})$} \\
\hline$<8$ & 71 & & \\
\hline$\geq 8$ & 29 & & \\
\hline \multicolumn{4}{|c|}{ Clinical stage (n) } \\
\hline IIA & 31 & & \\
\hline IIB/III & 69 & & \\
\hline \multicolumn{4}{|l|}{ Tumor grade (n) } \\
\hline Low & 39 & & \\
\hline High & 61 & & \\
\hline \multicolumn{4}{|l|}{ Metastasis (n) } \\
\hline With & 65 & & \\
\hline Without & 35 & & \\
\hline \multicolumn{4}{|c|}{ Chemotherapy (n) } \\
\hline Poor & 63 & & \\
\hline Good & 37 & & \\
\hline
\end{tabular}

those with benign bone tumors (Fig. 3B). As shown in Fig. 3C, circulating MIC-1 may assist in differentiating patients with late-stage cancer, for example stage III, from those with early-stage cancer, for example stage IIA. The aforementioned findings indicated that circulating MIC-1 levels also assist in distinguish among different stages of osteosarcoma; therefore, MIC-1 may serve as a potential biomarker of disease progression.

Correlation between circulating MIC-1 and osteosarcoma prognosis. All 100 patients with osteosarcoma included in the 


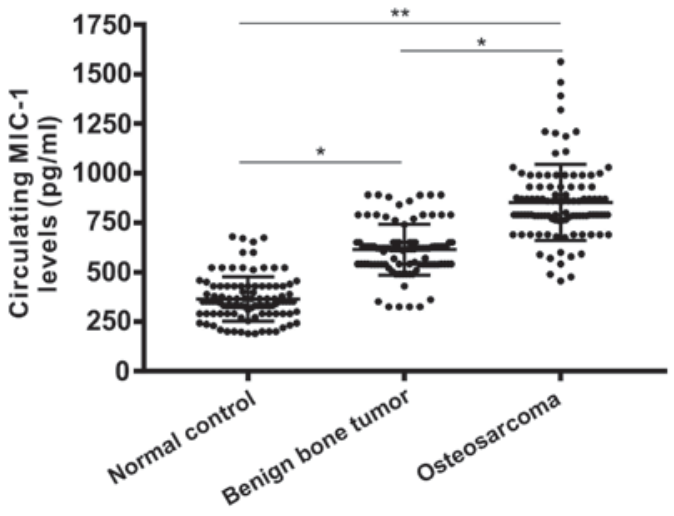

Figure 1. Circulating MIC-1 levels among healthy controls, patients with benign bone tumors, and patients with osteosarcoma were analyzed by enzyme-linked immunosorbent assays. ${ }^{*} \mathrm{P}<0.05$; ${ }^{* *} \mathrm{P}<0.01$. MIC- 1 , macrophage inhibitory cytokine 1 .

present study received a complete set of unified treatments and complete follow-up evaluation. On categorizing patients with osteosarcoma in accordance with average circulating levels of MIC-1, two types of patient group were obtained: High and low. Significant differences in overall survival rate were detected between the high circulating MIC-1 expression group and the low MIC-1 expression group (Fig. 4). Patients with high circulating MIC-1 levels tended to have a shorter survival rate, compared with those with low circulating MIC-1 levels. To determine whether MIC-1 is a potential prognostic indicator of osteosarcoma, Cox's univariate and multivariate regression analyses were performed, which suggested that a high level of MIC-1 served as a functional biomarker in the poor prognosis group (Table III).

MIC-1 levels in osteosarcoma tumor tissue. The levels of MIC-1 in tumor tissue and adjacent tissue were detected via RT-qPCR analysis. As shown in Fig. 5A, the mRNA levels of MIC-1 were considerably higher in the osteosarcoma tumor tissue than in the tumor-adjacent tissue. Concurrently, western blot analysis revealed an enhancement in the protein levels of MIC-1 in osteosarcoma cancer tissues (Fig. 5B). These data suggested that the increased production of MIC-1 may be a potential source of circulating MIC-1.

\section{Discussion}

Osteosarcoma is a popular primary bone malignancy predominantly occurring in teenagers, infants, and adults $(15,16)$. Osteosarcoma usually occurs in the metaphysis of long bones; however, it can also occur in the proximal tibia and distal femur (16). For patients with osteosarcoma with no metastases upon initial diagnosis, surgery, radiation therapy and chemotherapy considerably increased a patients' 5-year survival rate (1). However, a large proportion of patients are diagnosed with osteosarcoma at a late stage. For these individuals, a short survival rate is common $(1,17)$. Due to the low incidence and marked heterogeneity of osteosarcoma, its pathological molecular mechanism is less clear than that of other types of tumor (18). Currently, neoadjuvant chemotherapy with ifosfamide, cisplatin, methotrexate, and doxorubicin is frequently applied in the treatment of patients with osteosarcoma to prolong survival rate and suppress recurrence $(1,19)$. Identifying diagnostic tumor biomarkers for the early detection of metastases and survival prediction is of interest in investigations.

MIC-1 is a dissimilative member of the TGF- $\beta$ family, which can be a potential therapeutic target and/or a prognostic biological indicator for the treatment of certain tumor types $(20,21)$. Until now, no studies have reported the association between the levels of MIC-1 and factors associated with osteosarcoma. Therefore, the present study assessed MIC-1 levels in the context of osteosarcoma and observed that the mRNA and protein levels of MIC-1 were considerably elevated in osteosarcoma tissues, compared with those in tumor-adjacent tissues. The role of MIC-1 in diagnosis and prognosis was assessed, as a potentially nonintrusive serological biological marker, and it was revealed that the level of circulating MIC-1 was markedly elevated in patients with osteosarcoma and that this level was closely associated with a low cancer grade, late cancer stage and distant metastases. The circulating and tissue levels of MIC-1 may facilitate the diagnosis in differentiating patients with osteosarcoma from those with benign bone tumors, and for early-stage and late-stage diagnoses of osteosarcoma. In addition, increased circulating MIC-1 levels can serve as an independent biological marker in prognosis of osteosarcoma.

The present study is the first, to the best of our knowledge, to reveal the value of MIC-1 as a diagnostic biomarker for osteosarcoma. Furthermore, analysis of circulating MIC-1 in patients with osteosarcoma accurately differentiated cancerous lesions from noncancerous lesions. The results of the present study are concurrent with previous findings wherein circulating MIC-1 was associated with cancer. Based on previous studies, the diagnostic efficiency of circulating MIC-1 varies among different types of cancer; however, as an independent diagnostic biomarker, large AUCs of circulating MIC-1 have been observed among patients with pancreatic carcinoma and non-small-cell lung cancer (22-24). The ROC figures corroborated the weak ability of MIC-1 to distinguish osteosarcoma patients from those with noncancerous tumors and healthy controls. Other serological markers also show similar weak sensitivity, and their elevation is currently a clinical challenge. Previous studies have reported that advances in immune bead assessment and protein microarrays facilitate the concurrent identification of various serologic biological markers, which may contribute to resolving the issue of sensitivity (25-27).

The present study revealed a strong correlation between levels of MIC-1 and the survival rate of patients with osteosarcoma. The results suggested that higher levels of MIC-1 increased the mortality rate, compared with lower levels of MIC-1. Various elements can influence the prognosis of patients with osteosarcoma, including demographic factors, the size and position of tumor, tumor stage, and patients' response to chemotherapy. The present study showed that circulating MIC-1 was positively correlated with the prognosis of osteosarcoma. In addition, tumor grade and stage, reaction to chemotherapy, and distant metastases were associated with its prognosis. Circulating MIC-1 levels were measured in a blinded manner and ELISA was performed as 

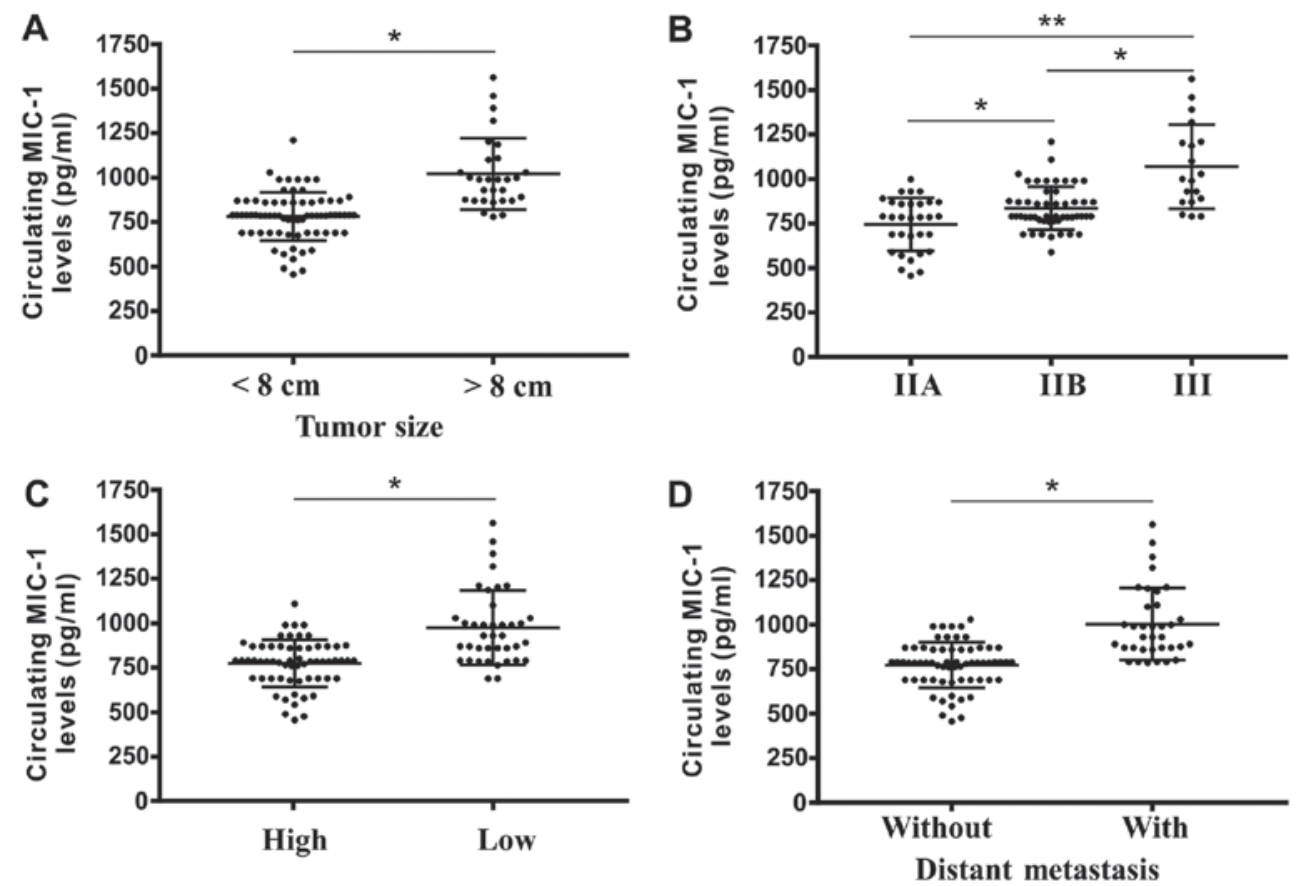

Figure 2. Correlation between the level of circulating MIC-1 and features of patients with osteosarcoma. Circulating MIC-1 levels in patients with osteosarcoma with (A) differently sized tumors, (B) tumor stages, (C) tumor grades, and the (D) presence/absence of distant metastases. ${ }^{*} \mathrm{P}<0.05$; ${ }^{* *} \mathrm{P}<0.01$. MIC-1, macrophage inhibitory cytokine 1 .
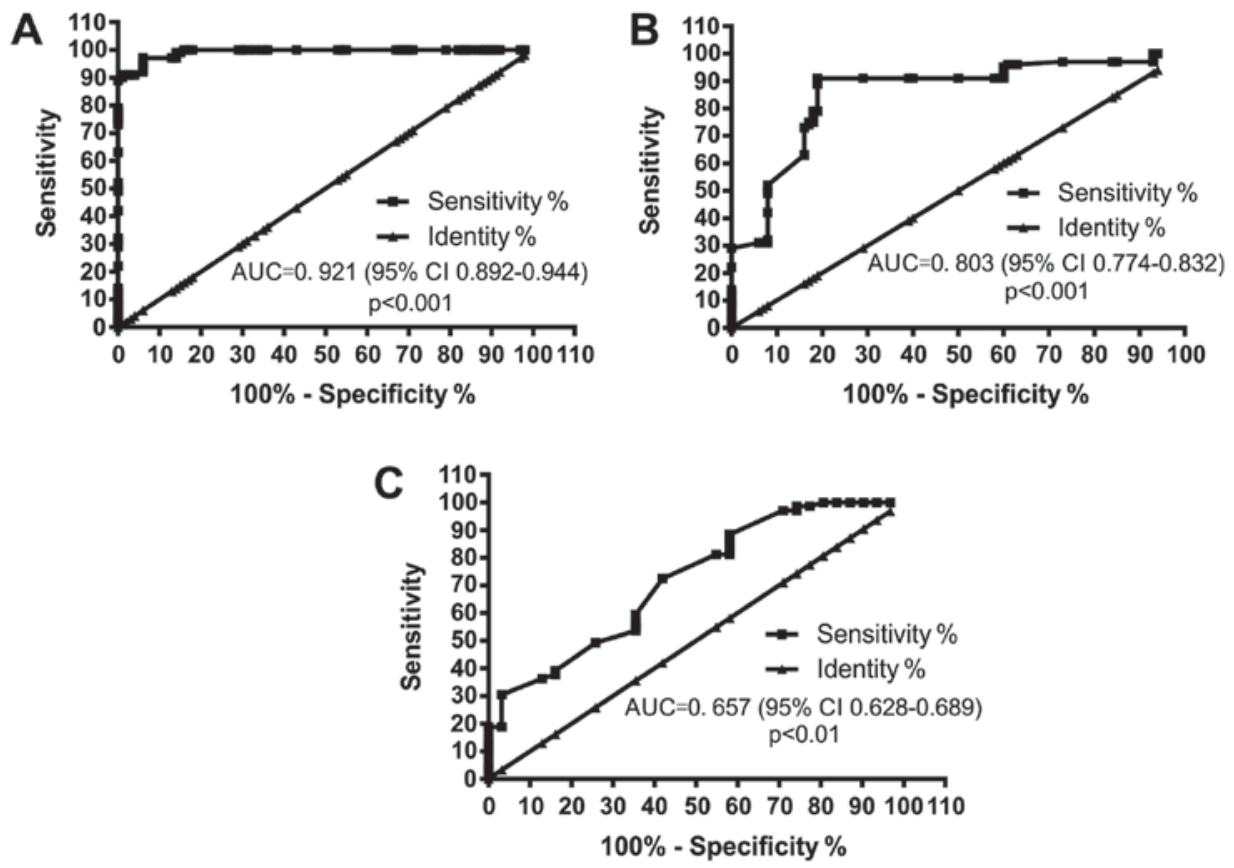

Figure 3. ROC assay of circulating MIC-1 in differentiating patients with osteosarcoma from those with noncancerous bone tumors and healthy controls. ROC of circulating MIC-1 levels differentiating patients with osteosarcoma from (A) healthy controls and (B) patients with noncancerous bone tumor and in (C) screening patients with IIB or III stage osteosarcoma from those with IIA stage osteosarcoma. ROC, receiving operating characteristic; AUC, area under the ROC curve; MIC-1, macrophage inhibitory cytokine 1.

a quantitative method to assess circulating MIC-1. The use of ELISA is advantageous as the procedure is fairly simple and is easily replicable, thereby increasing the reliability of results. However, the present study has the following limitations. Owing to the inter-session arrangement, the correlation between circulating MIC-1 levels and risk of osteosarcoma was not assessed. Furthermore, the specific source of higher circulating MIC-1 levels was unknown. Higher MIC-1 concentrations were noted in osteosarcoma tissues; however, it is unknown whether the increased levels of circulating MIC-1 were completely attributable to the overexpression of MIC-1 in osteosarcoma tissue. Further thorough investigations are 
Table III. Univariate and multivariate Cox proportional hazard analysis for overall survival rate of patients with osteosarcoma.

\begin{tabular}{|c|c|c|c|c|}
\hline \multirow[b]{2}{*}{ Factor (category) } & \multicolumn{2}{|c|}{ Univariate } & \multicolumn{2}{|c|}{ Multivariate } \\
\hline & HR $(95 \% \mathrm{CI})$ & $\mathrm{P}$-value & HR $(95 \% \mathrm{CI})$ & P-value \\
\hline Age $(\geq 20 /<20$ years $)$ & $0.43(0.21-0.96)$ & 0.75 & & \\
\hline Gender (male/female) & $1.01(0.49-2.04)$ & 0.31 & & \\
\hline Tumor size $(\geq 8 /<8 \mathrm{~cm})$ & $1.44(0.65-2.65)$ & 0.11 & & \\
\hline Tumor location (femur + tibia/other) & $1.32(0.54-2.21)$ & 0.21 & & \\
\hline Tim-3 level (high/low) & $2.98(2.54-3.43)$ & $<0.01$ & $1.98(1.52-2.86)$ & $<0.01$ \\
\hline Tumor stage (IIB+III/IIA) & $4.17(2.84-6.13)$ & $<0.05$ & $2.71(1.25-3.88)$ & $<0.01$ \\
\hline Distant metastases (yes/no) & $5.23(3.67-8.57)$ & $<0.05$ & $4.12(2.55-6.24)$ & $<0.01$ \\
\hline Chemotherapy response (poor/good) & $3.78(1.78-4.89)$ & $<0.01$ & $2.75(2.21-4.01)$ & $<0.01$ \\
\hline Tumor grade (low/high) & $2.63(1.61-3.75)$ & $<0.01$ & $2.19(1.01-3.14)$ & $<0.01$ \\
\hline
\end{tabular}

HR, hazard ratio; CI, confidence interval.

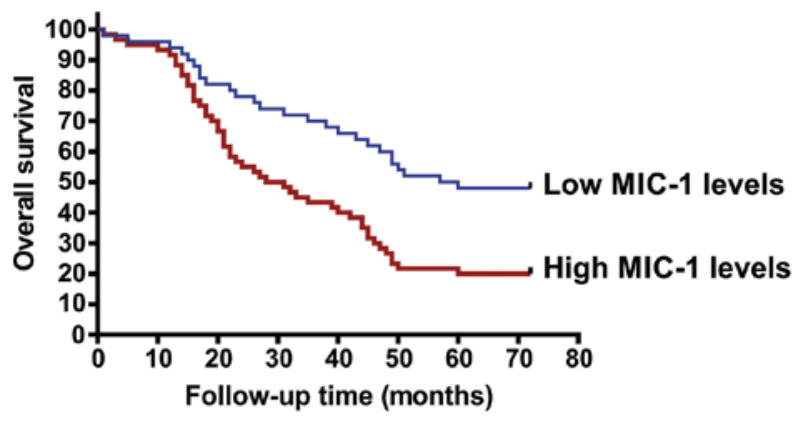

Figure 4. Correlation between MIC-1 levels and survival rate. Kaplan-Meier analysis of overall survival rate of patients with osteosarcoma with increased levels of circulating MIC-1 in comparison with those with low MIC-1 levels. MIC-1, macrophage inhibitory cytokine 1 .

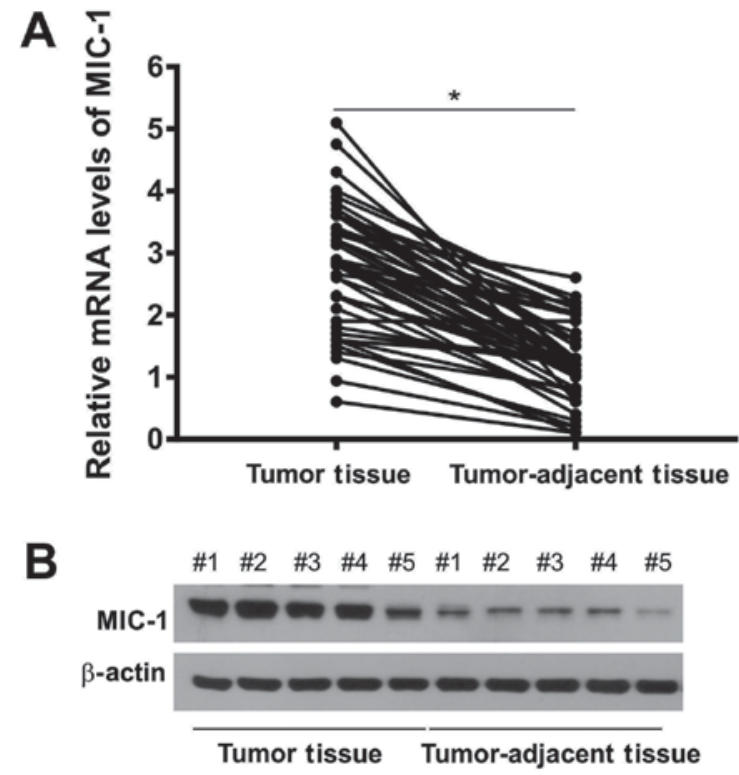

Figure 5. Expression of MIC-1 in osteosarcoma tissue. (A) mRNA level of MIC-1 in cancer tissues and adjacent tissues were analyzed by reverse transcription-quantitative polymerase chain reaction analysis $\left({ }^{*} \mathrm{P}<0.05\right)$. (B) Protein levels of MIC-1 in cancer tissues and adjacent tissues was detected by western blot analysis. MIC-1, macrophage inhibitory cytokine 1 . \#1-\#5 are the number of tumor or tumor-adjcent tissues. required to determine the reason underlying the increase in MIC-1 levels.

In conclusion, the present study revealed that MIC-1 was an efficient potential diagnostic biological indicator in patients with osteosarcoma. It was also demonstrated that circulating MIC-1 levels were usually elevated among patients with osteosarcoma with distant metastasis in relatively late clinical stages, in comparison with those who did not have distant metastasis or an advanced tumor stage. Multivariate survival analyses verified that circulating MIC-1 was an independent prognostic indicator for overall survival rate among patients with osteosarcoma. These findings bear testimony for the possible utility of circulating MIC- 1 as a potential noninvasive screening and prognostic indicator of osteosarcoma.

\section{Acknowledgements}

Not applicable.

\section{Funding}

No funding was received.

\section{Availability of data and materials}

All data generated or analyzed during this study are included in this published article.

\section{Authors' contributions}

XS conceived and designed the experiments; LT and YQ performed the experiments; DS analyzed the data; XS and LZ contributed reagents/materials/analysis tools; and XS wrote the manuscript.

\section{Ethics approval and consent to participate}

The experiments and all follow-up evaluations gained approval from the Ethics Committee of Xuzhou Children's Hospital 
(no. 20130116) and all participants provided written informed consent.

\section{Patient consent for publication}

Not applicable.

\section{Competing interests}

The authors declare that they have no competing interests.

\section{References}

1. Geller DS and Gorlick R: Osteosarcoma: A review of diagnosis, management, and treatment strategies. Clin Adv Hematol Oncol 8: 705-718, 2010

2. Taran SJ, Taran R and Malipatil NB: Pediatric osteosarcoma: An updated review. Indian J Med Paediatr Oncol 38: 33-43, 2017.

3. Davis AM, Bell RS and Goodwin PJ: Prognostic factors in osteosarcoma: A critical review. J Clin Oncol 12: 423-431, 1994.

4. Shankar GM, Clarke MJ, Ailon T, Rhines LD, Patel SR, Sahgal A, Laufer I, Chou D, Bilsky MH, Sciubba DM, et al: The role of revision surgery and adjuvant therapy following subtotal resection of osteosarcoma of the spine: A systematic review with meta-analysis. J Neurosurg Spine 27: 97-104, 2017.

5. Bishop MW, Janeway KA and Gorlick R: Future directions in the treatment of osteosarcoma. Curr Opin Pediatr 28: 26-33, 2016.

6. Wan J, Zhang X, Liu T and Zhang X: Strategies and developments of immunotherapies in osteosarcoma. Oncol Lett 11: 511-520, 2016

7. Hansen MF, Seton M and Merchant A: Osteosarcoma in Paget's disease of bone. J Bone Miner Res 21 (Suppl 2): P58-P63, 2006.

8. Chakravarty K, Saeed IT and Fowler RW: A fatal case of pleural osteosarcoma mimicking mesothelioma in Paget's disease of bone. Rheumatology (Oxford) 42: 1578-1579, 2003.

9. Khaled YS, Elkord E and Ammori BJ: Macrophage inhibitory cytokine-1: A review of its pleiotropic actions in cancer. Cancer Biomark 11: 183-190, 2012.

10. Jiang J, Wen W and Sachdev PS: Macrophage inhibitory cytokine-1/growth differentiation factor 15 as a marker of cognitive ageing and dementia. Curr Opin Psychiatry 29: 181-186, 2016.

11. Fuchs T, Trollor JN, Crawford J, Brown DA, Baune BT, Samaras K, Campbell L, Breit SN, Brodaty H, Sachdev P, et al: Macrophage inhibitory cytokine-1 is associated with cognitive impairment and predicts cognitive decline-the Sydney memory and aging study. Aging Cell 12: 882-889, 2013.

12. Yang H, Choi HJ, Park SH, Kim JS and Moon Y: Macrophage inhibitory cytokine-1 (MIC-1) and subsequent urokinase-type plasminogen activator mediate cell death responses by ribotoxic anisomycin in HCT-116 colon cancer cells. Biochem Pharmacol 78: 1205-1213, 2009.
13. Brown DA, Hance KW, Rogers CJ, Sansbury LB, Albert PS, Murphy G, Laiyemo AO, Wang Z, Cross AJ, Schatzkin A, et al: Serum macrophage inhibitory cytokine-1 (MIC-1/GDF15): A potential screening tool for the prevention of colon cancer? Cancer Epidemiol Biomarkers Prev 21: 337-346, 2012.

14. Livak KJ and Schmittgen TD: Analysis of relative gene expression data using real-time quantitative PCR and the 2(-Delta Delta C(T)) method. Methods 25: 402-408, 2001.

15. Broadhead ML, Clark JC, Myers DE, Dass CR and Choong PF: The molecular pathogenesis of osteosarcoma: A review. Sarcoma 2011: 959248, 2011.

16. He JP, Hao Y, Wang XL, Yang XJ, Shao JF, Guo FJ and Feng JX: Review of the molecular pathogenesis of osteosarcoma. Asian Pac J Cancer Prev 15: 5967-5976, 2014.

17. Isakoff MS, Bielack SS, Meltzer P and Gorlick R: Osteosarcoma: Current treatment and a collaborative pathway to success. J Clin Oncol 33: 3029-3035, 2015.

18. Marino-Enriquez A and Bovée JV: Molecular pathogenesis and diagnostic, prognostic and predictive molecular markers in sarcoma. Surg Pathol Clin 9: 457-473, 2016.

19. Durfee RA, Mohammed M and Luu HH: Review of osteosarcoma and current management. Rheumatol Ther 3: 221-243, 2016.

20. Unsicker K, Spittau B and Krieglstein K: The multiple facets of the TGF- $\beta$ family cytokine growth/differentiation factor-15/macrophage inhibitory cytokine-1. Cytokine Growth Factor Rev 24: 373-384, 2013.

21. Mimeault M and Batra SK: Divergent molecular mechanisms underlying the pleiotropic functions of macrophage inhibitory cytokine-1 in cancer. J Cell Physiol 224: 626-635, 2010.

22. Mohamed AA, Soliman H, Ismail M, Ziada D, Farid TM, Aref AM, Al Daly ME and Abd Elmageed ZY: Evaluation of circulating ADH and MIC-1 as diagnostic markers in Egyptian patients with pancreatic cancer. Pancreatology 15: 34-39, 2015.

23. Wang X, Li Y, Tian H, Qi J, Li M, Fu C, Wu F, Wang Y, Cheng D, Zhao W, et al: Macrophage inhibitory cytokine 1 (MIC-1/GDF15) as a novel diagnostic serum biomarker in pancreatic ductal adenocarcinoma. BMC Cancer 14: 578, 2014.

24. Liu YN, Wang XB, Wang T, Zhang C, Zhang KP, Zhi XY, Zhang W and Sun KL: Macrophage inhibitory Cytokine-1 as a novel diagnostic and prognostic biomarker in stage I and II nonsmall cell lung cancer. Chin Med J (Engl) 129: 2026-2032, 2016.

25. Zhou F, Xu X, Wu S, Cui X, Fan L and Pan W: Protein array identification of protein markers for serodiagnosis of Mycobacterium tuberculosis infection. Sci Rep 5: 15349, 2015.

26. Anderson KS, Sibani S, Wallstrom G, Qiu J, Mendoza EA, Raphael J, Hainsworth E, Montor WR, Wong J, Park JG, et al: Protein microarray signature of autoantibody biomarkers for the early detection of breast cancer. J Proteome Res 10: 85-96, 2011.

27. Aziz N: Measurement of circulating cytokines and immune-activation markers by multiplex technology in the clinical setting: What are we really measuring? For Immunopathol Dis Therap 6: 19-22, 2015. 